\title{
An Efficient Projector-Based Passivity Test for Descriptor Systems
}

\author{
Zheng Zhang, Student Member, IEEE, and Ngai Wong, Member, IEEE
}

\begin{abstract}
An efficient passivity test based on canonical projector techniques is proposed for descriptor systems (DSs) widely encountered in circuit and system modeling. The test features a natural flow that first evaluates the index of a DS, followed by possible decoupling into its proper and improper subsystems. Explicit state-space formulations for respective subsystems are derived to facilitate further processing such as model order reduction and/or passivity enforcement. Efficient projector construction and a fast generalized Hamiltonian test for the properpart passivity are also elaborated. Numerical examples then confirm the superiority of the proposed method over existing passivity tests for DSs based on linear matrix inequalities or skew-Hamiltonian/Hamiltonian matrix pencils.
\end{abstract}

Index Terms-Canonical projector, descriptor system (DS), generalized Hamiltonian, passivity.

\section{INTRODUCTION}

$\mathbf{P}$ ASSIVITY of a linear system is a crucial property for ensuring the stability of global simulation [1]-[3], namely, when the system is connected to other models for a full-system simulation. A passive system is one that does not generate energy internally, and a strictly passive system is a dissipative system. In circuit and multibody dynamics macromodeling, models are often casted as differential-algebraic equations [4][7] known also as singular systems or descriptor systems (DSs) [8]-[10]. Specifically, DSs encompass a larger set with much higher modeling flexibility than the more restrictive standard state space systems. In fact, a major use of DSs is in the modified nodal analysis (MNA) [6], [7], [11] which describes a generally multi-port electrical circuit as a DS. Electromagnetic simulations of on-chip structures normally yield DS models [12]. Moreover, the Loewner matrix interpolation technique [13], [14] has also been advocated recently to fit measured/simulated data of electronic circuits/systems to produce the corresponding DS. Such framework is superior to the traditional vector fitting (which results in regular state spaces) in the sense that no manual pole initialization is needed and

Manuscript received June 23, 2009; revised December 11, 2009. Date of current version July 21, 2010. This work was supported in part by the Hong Kong Research Grants Council, under Project HKU 718509E, and the University Research Committee of the University of Hong Kong. This paper was recommended by Associate Editor J. R. Phillips.

The authors are with the Department of Electrical and Electronic Engineering, University of Hong Kong, Pokfulam Road, Hong Kong (e-mail: zzhang@eee.hku.hk; nwong@eee.hku.hk).

Color versions of one or more of the figures in this paper are available online at http://ieeexplore.iee.org.

Digital Object Identifier 10.1109/TCAD.2010.2049048 that the optimal model order can be automatically extracted from the data [13], [14]. Other attempts have utilized DSs in the first-order macromodeling of second-order systems such as RLCK circuits where " $K$ " denotes susceptance [15]. Although the DS in [15] can still be reduced to a standard state space system, a DS representation preserves the structural stamps and facilitates fast computation through possible utilization of matrix structures and sparsity.

However, the passivity test for a DS, usually as a preprocessing step for verifying model validity, is far less developed than for its standard state space counterparts whose passivity can be readily checked by the positive real lemma or through frequency sweeping [16]-[18]. Extensions of the positive real lemma to DSs based on linear matrix inequalities (LMIs) and projected Lur'e matrix equations have been presented in [19] and [20], respectively. However, their numerical implementations result in prohibitive computation due to the expensive solution of LMIs. Although generalized Schur form approaches [21]-[23], and a skew-Hamiltonian/Hamiltonian (SHH) transformation [2] have been proposed for testing DS passivity with higher efficiency, these algorithms still require multiple expensive singular value decompositions (SVDs), Schur, and QR factorizations.

To overcome the computational hurdle, the emerging canonical projector technique [24]-[26] is revised and utilized to formulate a highly efficient DS passivity test. In short, the canonical projectors give rise to a spectral projector that provides a natural and conceptually simple way to decouple a DS into its proper (impulse-free) subsystem and improper (impulsive) subsystem, if any. Such decoupling is considered the major difficulty in the passivity tests for DSs [2] (this is different from the early work of Cauer [27] on passive systems based on matrix polynomial transfer functions where subsystem decomposition is trivial compared to the otherwise DS formulation here). A major difference that marks the efficiency of the proposed method over existing ones is the early knowledge of the matrix pencil index, which quickly manifests as a direct consequence of constructing the matrix projector chain. Given that an (infinite) minimal passive system is of index at most two, the test quickly screens out nonpassive systems after two initial matrix chain iterations. Then, depending on the need, the proper and improper parts can be completely decoupled via spectral projectors and, respectively, tested for passivity.

A conference version of this paper is in [1], which reports preliminary results and standard SVD implementation for the 
DS passivity test. In this paper, we enrich the theoretical illustration, waive the stable matrix pencil assumption, and introduce a novel LU-based construction of projectors and an efficient implementation of the recent generalized Hamiltonian method [28], [29] for testing the passivity of the proper part. Such combination has made the proposed test truly applicable to large-scale practical DSs, as demonstrated in the much richer numerical section in this paper wherein the model sizes are larger than those in [1] by several orders. We also present a concise complexity analysis of existing DS passivity tests (in addition to those considered in [1]), which in turn confirms the superiority of the projector approach.

The organization of this paper is as follows. Section II reviews the problem setting and basics of passivity. Section III introduces the matrix chain theory and canonical/spectral projectors in a constructive and much clearer manner than the presentation in [25]. Section IV explicitly constructs the state spaces for the extracted proper and improper subsystems of a DS, upon which the passivity can be easily checked. Section V presents efficient numerical implementations for the proposed DS passivity test and discusses the limitations of existing tests. Numerical examples in Section VI then verify the efficiency of the proposed test, followed by some remarks in Section VII. Finally, Section VIII draws the conclusion.

\section{BASICS OF PASSIVITY}

We study a LTI DS in the form

$$
\begin{aligned}
E \dot{x} & =A x+B u \\
y & =C x+D u
\end{aligned}
$$

where $E, A \in \mathbb{R}^{n \times n}$ and $B, C^{T} \in \mathbb{R}^{n \times m}$. Also, $u, y \in \mathbb{R}^{m}$ and $x \in \mathbb{R}^{n}$ are the input, output, and state vectors, respectively. The matrix $E$ is generally singular with $\operatorname{rank}(E) \leq n$. We assume that the matrix pencil $\lambda E-A$ is regular, i.e., $\operatorname{det}\left(\lambda_{0} E-A\right) \neq 0$ for some $\lambda_{0} \in \mathbb{C}$. Then, there always exist nonsingular $W, T \in \mathbb{R}^{n \times n}$ that transform $E$ and $A$ into the so-called Weierstrass canonical form [24]

$$
E=W\left[\begin{array}{cc}
I_{q} & 0 \\
0 & N
\end{array}\right] T, \quad A=W\left[\begin{array}{cc}
J & 0 \\
0 & I_{n-q}
\end{array}\right] T
$$

where $I_{k}$ denotes an identity matrix of order $k$ (though the dimension is sometimes omitted when it is clear from context). The matrix $J$ corresponds to the finite eigenvalues of $\lambda E-A$, whereas $N$ is nilpotent and corresponds to the infinite eigenvalues. When all eigenvalues of $J$ have negative real parts, the pencil $\lambda E-A$ is said to be stable. The nilpotency index $\mu$ of $N$, viz. $N^{\mu-1} \neq 0$ and $N^{\mu}=0$, is called the index of the matrix pencil $\lambda E-A$.

Referring to the Weierstrass canonical form (2), we define the left and right (spectral) projectors, $P_{l}$ and $P_{r}$, respectively, as

$$
P_{l}=W\left[\begin{array}{cc}
I_{q} & 0 \\
0 & 0
\end{array}\right] W^{-1}, \quad P_{r}=T^{-1}\left[\begin{array}{cc}
I_{q} & 0 \\
0 & 0
\end{array}\right] T .
$$

Obviously, $P_{l}$ and $P_{r}$ are the projectors onto the left and right deflating subspaces, respectively, corresponding to the finite eigenvalues along the left and right deflating subspaces corresponding to the eigenvalue at infinity, whereas $Q_{l}=I-P_{l}$ and $Q_{r}=I-P_{r}$ are the complementary projectors.

Using the Weierstrass canonical form (2) and partitioning $\left[\begin{array}{ll}C_{p} & C_{\infty}\end{array}\right]=C T^{-1}$ and $\left[\begin{array}{ll}B_{p}^{T} & B_{\infty}^{T}\end{array}\right]^{T}=W^{-1} B$ conformal to (2), the DS transfer function $G(s)=D+C(s E-A)^{-1} B$ of (1) can be expressed as

$$
\begin{aligned}
& G(s)=D+C(s E-A)^{-1} B \\
& =D+\left[\begin{array}{ll}
C_{p} & C_{\infty}
\end{array}\right]\left[\begin{array}{c}
\left(s I_{q}-J\right)^{-1}-\left(I_{n-q}-s N\right)^{-1}
\end{array}\right]\left[\begin{array}{c}
B_{p} \\
B_{\infty}
\end{array}\right] \\
& =\underbrace{D-C_{\infty} B_{\infty}+C_{p}\left(s I_{q}-J\right)^{-1} B_{p}}_{G_{p}(s)} \\
& \underbrace{-s C_{\infty} N B_{\infty}-s^{2} C_{\infty} N^{2} B_{\infty}-s^{3} C_{\infty} N^{3} B_{\infty}-\cdots}_{G_{\infty}(s)}
\end{aligned}
$$

where $G_{p}(s)$ is the proper part (bounded as $s \rightarrow \infty$ ) and $G_{\infty}(s)$ the improper part (unbounded as $s \rightarrow \infty$ ) of $G(s)$.

For an LTI system, (strict) passivity is equivalent to the transfer function being (strictly) positive real.

Theorem 1 (See [19]): A rational matrix-valued transfer function $G(s) \in \mathbb{C}^{m \times m}$ is positive real (strictly positive real) if:

1) $G(s)$ is analytic in $\mathbb{C}_{+}=\{s \in \mathbb{C} \mid \operatorname{Re}(s)>0\}$;

2) $\Phi(j \omega)=G(j \omega)+(G(j \omega))^{H}$ is positive semidefinite (positive definite) for all $\omega \in \mathbb{R}$ such that $j \omega$ is not a pole of $G(s)$;

3) j$j \omega_{0}$ or $\infty$ is a pole of $G(s)$, then it is a simple pole and the $m \times m$ residue matrix is positive semidefinite.

Based on Theorem 1, if $G(s)$ in (4) is viewed as

$$
G(s)=\overbrace{\text { strictly proper }}^{\underbrace{G_{s p}(s)}_{s p}+M_{0}}+\underbrace{s M_{1}+\sum_{k=2}^{\infty} s^{k} M_{k}}_{G_{\infty}(s)}
$$

then $G(s)$ is positive real if and only if $G_{p}(s)$ is positive real, $M_{1} \geq 0$ and $M_{k}=0$ for $k \geq 2$. Comparing (5) and (4), it is obvious that (1) is passive if and only if $G_{p}(s)$ in (4) is passive and $M_{1}=-C_{\infty} N B_{\infty} \geq 0$, whereas $C_{\infty} N^{i} B_{\infty}=0$, $i=2,3, \cdots$. Consequently, a key to testing the passivity of a DS is to first decouple it into its proper and improper parts.

\section{MATRIX PRojector Chain}

Setting $E_{0}:=E$ and $A_{0}:=A$, we consider a matrix chain

$$
E_{j+1}:=E_{j}+A_{j} Q_{j}, \quad A_{j+1}:=A_{j} P_{j}
$$

where $Q_{j}$ is a projector onto $\operatorname{ker} E_{j}$, i.e., $Q_{j}^{2}=Q_{j}$ and $\operatorname{im} Q_{j}=$ $\operatorname{ker} E_{j}$, and $P_{j}=I-Q_{j}$ is a projector along $\operatorname{ker} E_{j}$ for $j=$ $0,1, \ldots$ Obviously, $P_{j} Q_{j}=Q_{j} P_{j}=0$. The following theorem establishes the properties of the matrices $E_{j}$ 's.

Theorem 2 (See [24]): For a regular index- $\mu$ pencil $\lambda E-$ $A$, the matrices $E_{0}, \cdots, E_{\mu-1}$ are singular, while $E_{\mu}$ is nonsingular.

Consequently, for the matrix chain $\left\{E_{j}, A_{j}\right\}$ we have the following.

1) If $E_{1}$ is nonsingular, i.e., $\mu=1$ or $N=0$, then system (1) is impulse-free [2]. In this case, a standard state space 
system can be extracted and tested for passivity, see Section IV-A.

2) If $E_{1}$ is singular but $E_{2}$ is nonsingular, we then proceed to form the spectral projector $P_{r}$ (or $P_{l}$ ) through constructing canonical projectors, which allows the decoupling of $G(s)$ into the proper and improper parts $G_{p}(s)$ and $G_{\infty}(s)$, respectively.

3) If $E_{2}$ is singular, then (infinite) minimality of the DS dictates $M_{k} \neq 0$ for $k \geq 2$ [19] (see also remark 3 in Section VII), so the system is nonpassive and the passivity test is complete.

Next, we present some important properties of the matrix chain.

1) Post-multiplying the left equation in (6) by $Q_{j}$ and $P_{j}$, respectively, yields

$$
\begin{aligned}
& E_{j+1} Q_{j}=A_{j} Q_{j} \\
& E_{j+1} P_{j}=E_{j}
\end{aligned}
$$

for $j=0,1, \cdots, \mu-1$.

2) The projectors $Q_{0}, \cdots, Q_{\mu-1}$ are called admissible if they satisfy $Q_{j} Q_{i}=0$ for $j>i$. Many new properties arise from using admissible projectors. For example, for $j=0, \cdots, \mu-1$, we have

$$
\begin{aligned}
E_{\mu} Q_{j} & =A_{j} Q_{j} \\
A_{j+1} & =A-E_{\mu}\left(Q_{0}+\cdots+Q_{j}\right) .
\end{aligned}
$$

3) Canonical projectors are admissible projectors that further satisfy

$$
\begin{aligned}
Q_{j}= & Q_{j} P_{j+1} \cdots P_{\mu-1} E_{\mu}^{-1} A_{j} \\
& =Q_{j} P_{j+1} \cdots P_{\mu-1} E_{\mu}^{-1} A
\end{aligned}
$$

for $j=0, \cdots, \mu-2$, and

$$
Q_{\mu-1}=Q_{\mu-1} E_{\mu}^{-1} A_{\mu-1}=Q_{\mu-1} E_{\mu}^{-1} A .
$$

The second equality signs in (9) and (10) can be established from (8b).

4) In the matrix chain (6), the projector $Q_{j}$ is, generally, not unique as only its range is constrained. Suppose that $\bar{Q}_{j}=\bar{Q}_{j}^{2}$ is another projector onto ker $E_{j}$. Then, $\bar{Q}_{j} Q_{j}=$ $Q_{j}, Q_{j} \bar{Q}_{j}=\bar{Q}_{j}$, and $-\bar{Q}_{j} P_{j}=Q_{j} \bar{P}_{j}$. This permits a relationship between the $E_{j+1}$ 's generated by different projectors. We have

$$
\begin{aligned}
\bar{E}_{j+1} & =E_{j}+A_{j} Q_{j} \bar{Q}_{j}=E_{j}+A_{j} Q_{j}\left(I-\bar{P}_{j}\right) \\
& =\left(E_{j}+A_{j} Q_{j}\right)\left(I+\bar{Q}_{j} P_{j}\right) \\
& =E_{j+1}\left(I+\bar{Q}_{j} P_{j}\right) .
\end{aligned}
$$

Provided $E_{j+1}$ is invertible, the inverse of $\bar{E}_{j+1}$ is easily shown to be $\bar{E}_{j+1}^{-1}=\left(I-\bar{Q}_{j} P_{j}\right) E_{j+1}^{-1}$.

The following depicts the formation of canonical and spectral projectors for the non-trivial case $\mu=2$. Compared to [25], our presentation is highly straightforward and the proofs in the appendices are either new or much more elegant.

\section{A. Constructing Canonical Projectors}

Setting $E_{0}:=E$ and $A_{0}:=A$, the matrix chain in the case of $\mu=2$ takes the form

$$
\begin{aligned}
& E_{1}:=E_{0}+A_{0} Q_{0} \text { and } A_{1}:=A_{0} P_{0} \\
& E_{2}:=E_{1}+A_{1} Q_{1}
\end{aligned}
$$

where $E_{2}$ is nonsingular by Theorem 2 . The projectors $Q_{0}$ and $Q_{1}$ in (III-A) are, generally, neither canonical nor admissible, but a new matrix chain with canonical (and, therefore, admissible) projectors can be derived from (III-A) with careful reformulation, as illustrated in Appendix A. In short, the canonical projectors $Q_{1}^{\prime}$ and $Q_{0}^{\prime}$ can be computed as

$$
\begin{aligned}
Q_{1}^{\prime} & =Q_{1} E_{2}^{-1} A_{1} \\
Q_{0}^{\prime} & =Q_{0} P_{1}^{\prime} E_{2}^{\prime-1} A_{0}=Q_{0} P_{1}^{\prime}\left(I-Q_{1}^{\prime} P_{1}\right) E_{2}^{-1} A_{0} \\
& =Q_{0} P_{1}^{\prime} E_{2}^{-1} A_{0}=Q_{0}\left(I-Q_{1} E_{2}^{-1} A_{1}\right) E_{2}^{-1} A_{0} .
\end{aligned}
$$

\section{B. Constructing Spectral Projectors}

Following from above, an important result of canonical projectors is that the right spectral projector $P_{r}$ is readily given by

$$
P_{r}=P_{0}^{\prime} P_{1}^{\prime}=\left(I-Q_{0}^{\prime}\right)\left(I-Q_{1}^{\prime}\right) .
$$

The proof is shown in Appendix B. Using the expressions in (13) and (14) [or (47)], it can be shown that simple analytical spectral projectors are readily derived for some structured DSs [30], [31], thereby allowing fast spectral decomposition. Nonetheless, analytical projectors generally do not exist and specific numerical considerations are needed for practical spectral projector construction, as will be discussed in Section V-A.

\section{PAssivity Test}

The canonical projector technique provides a natural way to decouple the proper (impulse-free) and improper (impulsive) parts of the DS in (1), which translates into a highly effective way for checking passivity based on Theorem 1 and the conditions listed after (5). Moreover, all that is needed is one, either the left or right, spectral projector. Without loss of generality, we assume the availability of $P_{r}$ in (3). The case when $P_{l}$ is available follows analogously. In the following, we see how $P_{r}$ permits a simple and explicit construction of the proper and improper subsystems by the additive decomposition procedure.

\section{A. Additive Decomposition}

Assume that the proper part is realized by $G_{p}(s)=\tilde{C}(s \tilde{E}-$ $\tilde{A})^{-1} \tilde{B}+\tilde{D}$. With spectral projectors, the proper subsystem can be formed in various ways. By additive decomposition, a regular system can be constructed explicitly as

$$
\begin{aligned}
& \tilde{E}=E P_{r}-A\left(I-P_{r}\right), \tilde{A}=A \\
& \tilde{C}=C P_{r}, \tilde{B}=B, \tilde{D}=M_{0}
\end{aligned}
$$


where $M_{0}$ is readily obtained from (16) below. It is straightforward to prove that $\tilde{E}$ is nonsingular by this formulation, thus no further efforts (such as SVD) are needed to convert this DS to a regular one. To extract $G_{\infty}(s)$ in (4), we first construct an improper DS using the right spectral projector

$$
\begin{aligned}
G_{1}(s) & =D+C(I-P r)(s E-A)^{-1} B \\
& =D+C T^{-1}\left[\begin{array}{ll}
0 & \\
& I
\end{array}\right]\left[\begin{array}{rr}
s I-J & \\
& s N-I
\end{array}\right]^{-1} W^{-1} B \\
& =\underbrace{D-C_{\infty} B_{\infty}}_{M_{0}}-s C_{\infty} N B_{\infty}-s^{2} C_{\infty} N^{2} B_{\infty}-\cdots .
\end{aligned}
$$

Contrasting (4) and (5), it follows that $M_{1}=-C_{\infty} N B_{\infty}$ which can be shown to be

$$
\begin{aligned}
M_{1} & =-C A^{-1} E_{\infty} A^{-1} B \\
& =-C\left(E_{f}+A_{\infty}\right)^{-1} E_{\infty}\left(E_{f}+A_{\infty}\right)^{-1} B
\end{aligned}
$$

where $E_{f}:=E P_{r}$ and $A_{\infty}:=A\left(I-P_{r}\right)$. Subsequently, $M_{0}$ and $G_{p}(s)$ can be decided by (15) and (16). The merit of the alternative expression in (17b) is that the inverse of $\left(E_{f}+A_{\infty}\right)$ always exists despite the invertibility of $A$ [or equivalently that of $J$ in (2)]. The positive semidefiniteness of $M_{1}$ and $M_{0}$, necessary for a passive DS, can then be easily tested. In fact, $M_{1}$ and $M_{0}$ are normally small-size $m \times m$ square matrices since the number of input/output ports is usually much fewer than the state space dimension, i.e., $m \ll n$.

At the first glance, the passivity test for $G_{p}(s)$ can make use of the Hamiltonian matrix eigenvalue test for standard state space systems [16], since $\tilde{E}$ is nonsingular such that its inverse can be absorbed into $\tilde{A}$ and $\tilde{B}$ (though this is sometimes not preferable as $\tilde{E}$ may be ill-conditioned). For large systems, a fast numerical approach is developed [32] to compute the eigenvalues along the imaginary axis. However, Hamiltonianbased approach becomes computationally infeasible due to the expensive matrix inversion of $\tilde{E}$. Further, the matrix inversion also destroys the sparsity of system matrices, which further slows down the calculations. An alternative approach is to combine the fast eigenvalue solver of [32] with the recently proposed generalized Hamiltonian method (GHM [28], [29]), which is further elaborated in Section V-B. This method directly tests passivity on the DS-form proper subsystem without loss of sparsity, thus it is more efficient than using Hamiltonian method on the converted standard state-space model.

The flow of the proposed projector-based decoupled-DS passivity test is summarized in Fig. 1. A note about the infinite minimality assumption of the initial DS can be found in Section VII, remark 3.

\section{B. Reconstruction into a $D S$}

Finally, if $G_{p}(s)=\tilde{D}+\tilde{C}(s \tilde{E}-\tilde{A})^{-1} \tilde{B}$ is passive (possibly after passivity enforcement [16]) and $M_{1} \geq 0$, a passive DS corresponding to the transfer function $G_{p}(s)+s M_{1}$ can be reconstructed for export to a simulator. Indeed, let $M_{1}=Z Z^{T}$ be a Cholesky factorization, where $Z \in \mathbb{R}^{m \times m}$. Then, the DS

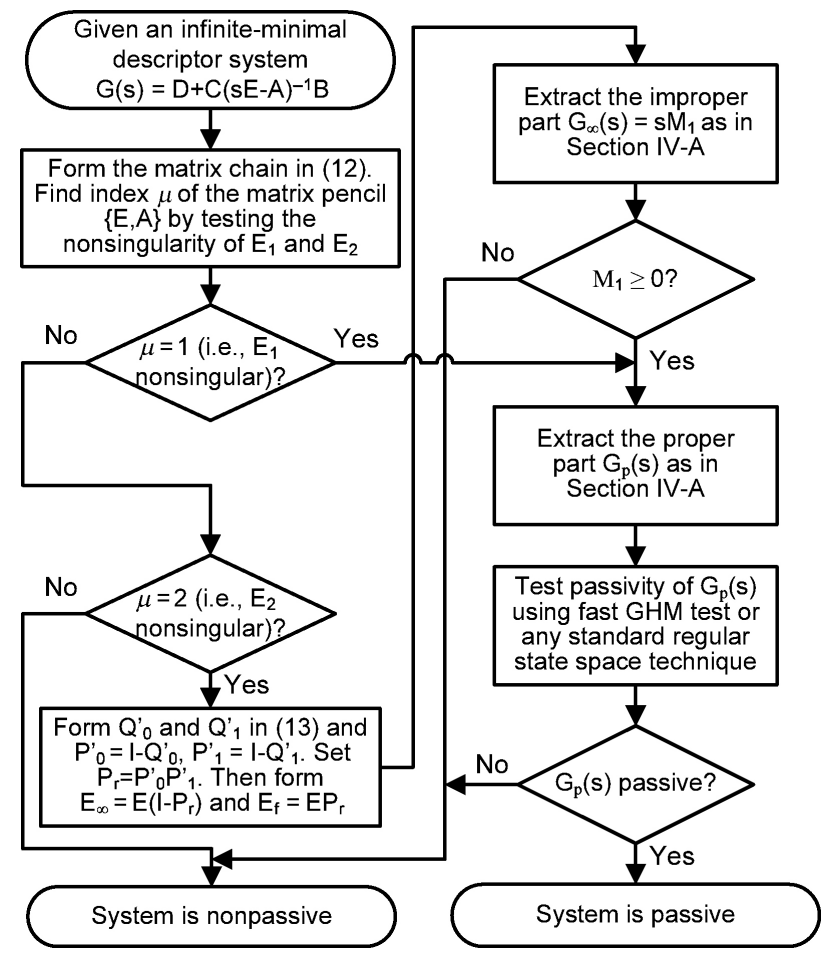

Fig. 1. Flowchart for the proposed DS passivity test in a pseudocodes style.

can be formulated as

$$
\begin{aligned}
{\left[\begin{array}{ccc}
\tilde{E} & & \\
& 0 & I_{m} \\
& 0 & 0
\end{array}\right] \dot{x} } & =\left[\begin{array}{ccc}
\tilde{A} & & \\
& I_{m} & 0 \\
& 0 & I_{m}
\end{array}\right] x+\left[\begin{array}{c}
\tilde{B} \\
0 \\
Z^{T}
\end{array}\right] u \\
y & =\left[\begin{array}{lll}
\tilde{C} & -Z & 0
\end{array}\right] x+\tilde{D} u
\end{aligned}
$$

whose transfer function is easily checked to be $G_{p}(s)+s M_{1}$.

\section{IMPLEMENTATION AND COMPLEXITY}

\section{A. Fast Spectral Projector Construction}

Although there exist closed-form spectral projectors in terms of the initial (not necessarily canonical) projectors as in (13) and (14), or for some special cases further simplified forms as in [30] and [31], the computation can still be expensive and constitute a bottleneck due to large-size nullspace identification, matrix multiplication and inverse. Here we elaborate an efficient numerical construction of spectral projectors, mainly by exploiting sparsity and low-rank matrix operations inherent to many physical problems. The illustration below is based on index-2 $(\mu=2)$ systems, and that for index-1 $(\mu=1)$ systems follows similarly.

First, a key step to forming the canonical projectors in (13) is to find the initial projectors $Q_{0}$ and $Q_{1}$ spanning the nullspaces of the usually sparse or structured $E_{0}$ and $E_{1}$. Standard ways of identifying the nullspace include SVD or alike, which do not utilize matrix patterns and can be expensive for large-size matrices. To this end, we employ the sparse LU decomposition-based routine from [33], called LUQ, which decomposes $E_{j}, j=0,1$, into

$$
E_{j}^{T}=L_{j}\left[\begin{array}{cc}
U_{j} & 0 \\
0 & 0
\end{array}\right] R_{j}
$$


where $L_{j}, R_{j} \in \mathbb{R}^{n \times n}$ are nonsingular matrices, $U_{j} \in \mathbb{R}^{r \times r}$ is a nonsingular upper triangular matrix, $r$ is the rank of $E_{j}$ and often $n-r \ll n$. Therefore, (19) is also used in our implementation for checking the nonsingularity of $E_{j}$. If $E_{j}$ is singular, its nullspace is then computed via the left nullspace of $E_{j}^{T}$ (solely due to the reason that $L_{j}$ is produced with higher numerical accuracy than $R_{j}$ by the LUQ routine [33]). For completeness, the LUQ routine is outlined in Appendix C. Obviously, using $I_{(r+1: n,:)}$ to denote the last $n-r$ rows of an $n \times$ $n$ identity matrix, the columns of $\left(I_{(r+1: n,:)} L_{j}^{-1}\right)^{T}$ span ker $E_{j}$. In practice, direct computation of this column matrix is not preferred as the condition number of $L_{j}$ can be large rendering ill-conditioned inverse. Instead, we perform a LU factorization of $L_{j}$ with permutation

$$
\tilde{P}_{j} L_{j}=\tilde{L}_{j} \tilde{U}_{j}
$$

where $\tilde{L}_{j}, \tilde{U}_{j}, \tilde{P}_{j} \in \mathbb{R}^{n \times n}$ such that $\tilde{P}_{j}$ is the permutation matrix with $\tilde{P}_{j}^{T} \tilde{P}_{j}=I, \tilde{U}_{j}$ is upper triangular and $\tilde{L}_{j}$ is lower triangular with unit diagonal entries and a condition number around unity. Then, it is easily checked that, due to the upper triangular structure of $\tilde{U}_{j},\left(I_{(r+1: n,:)} \tilde{L}_{j}^{-1} \tilde{P}_{j}\right)^{T}$ spans the same range as that by $\left(I_{(r+1: n,:)} L_{j}^{-1}\right)^{T}$ and has intrinsically better accuracy due to the low condition number of $\tilde{L}_{j}$. It is noted that computing $\tilde{L}_{j}$ does not really add to the computational load since the inverse of $L_{j}$ would generally proceed with an LU decomposition, of which $\tilde{L}_{j}$ is a byproduct, followed by backward/forward substitutions. Next, a (modified) Gram-Schmidt process, denoted by gs(o) below, leads to an orthonormal and usually thin column matrix, namely

$$
\Psi_{j}=\operatorname{gs}\left(\left(I_{(r+1: n,:)} \tilde{L}_{j}^{-1} \tilde{P}_{j}\right)^{T}\right)
$$

such that $Q_{j}=\Psi_{j} \Psi_{j}^{T}$ forms an orthogonal projector onto ker $E_{j}$. Subsequently, $Q_{0}=\Psi_{0} \Psi_{0}^{T}$ and $Q_{1}=\Psi_{1} \Psi_{1}^{T}$ are in low-rank factored forms that facilitate fast computation of canonical projectors in (13) by

$$
\begin{aligned}
& Q_{1}^{\prime}:=\Psi_{1}\left(\left(\Psi_{1}^{T} E_{2}^{-1}\right) A_{1}\right) \\
& Q_{0}^{\prime}:=\Psi_{0}\left(\left(\Psi_{0}^{T} P_{1}^{\prime} E_{2}^{-1}\right) A_{0}\right)
\end{aligned}
$$

which are again low-rank. Further computational savings can be achieved in (14) by recognizing that

$$
\begin{aligned}
Q_{0}^{\prime} Q_{1}^{\prime} & =Q_{0}^{\prime}\left(Q_{0}+P_{0}\right) Q_{1}^{\prime} \\
& =Q_{0} Q_{1}^{\prime}+Q_{0} P_{1}^{\prime} E_{2}^{\prime-1} A_{1} Q_{1}^{\prime} \\
& =Q_{0} Q_{1}^{\prime}+Q_{0} P_{1}^{\prime} E_{2}^{\prime-1} E_{2}^{\prime} Q_{1}^{\prime}=Q_{0} Q_{1}^{\prime}
\end{aligned}
$$

so that $P_{r}=I-Q_{0}^{\prime}-Q_{1}^{\prime}+Q_{0} Q_{1}^{\prime}$ where $Q_{0}^{\prime}, Q_{1}^{\prime}$, and $Q_{0} Q_{1}^{\prime}$ all involve low-rank factors only.

\section{B. Fast GHM Test for Proper-Part Passivity}

The spectral projector, with efficient implementation as above, allows fast decoupling of the proper and improper parts of a DS. However, the computational bottleneck for the DS passivity test still lies in testing the passivity of the proper part. To speed up the overall DS passivity test, having an efficient test for the proper part is important. For the DS-form proper subsystem constructed by (15), its passivity can be assessed by the matrix pencil $(\mathcal{J}, \mathcal{K})$ [28], [29]

$$
\begin{gathered}
\mathcal{J}=\left[\begin{array}{cc}
\tilde{A}-\tilde{B}\left(\tilde{D}+\tilde{D}^{T}\right)^{-1} C & -\tilde{B}\left(\tilde{D}+\tilde{D}^{T}\right)^{-1} \tilde{B}^{T} \\
\tilde{C}^{T}\left(\tilde{D}+\tilde{D}^{T}\right)^{-1} \tilde{C} & -\tilde{A}^{T}+\tilde{C}^{T}\left(\tilde{D}+\tilde{D}^{T}\right)^{-1} \tilde{B}^{T}
\end{array}\right] \\
\mathcal{K}=\left[\begin{array}{cc}
\tilde{E} & 0 \\
0 & \tilde{E}^{T}
\end{array}\right]
\end{gathered}
$$

any purely imaginary generalized eigenvalue of which pinpoints a crossover point of passivity violations. The proper part is strictly passive if and only if $\tilde{D}+\tilde{D}^{T}>0$ and $(\mathcal{J}, \mathcal{K})$ has no eigenvalues on the imaginary axis. This eigenvalue problem consumes an $O\left((2 n)^{3}\right)$ complexity, which is infeasible for large sparse system. In practice, the system matrices of $G_{p}(s)$ are normally sparse, and only results close to the imaginary axis are wanted for passivity verification. Based on this observation, the fast passivity characterization of [32] exclusive to large sparse state-space models can be extended to the DS case in (15).

To outline the fast Hamiltonian passivity test [32], we first assume the proper subsystem is a standard state-space model. In this case, $\operatorname{eig}(\mathcal{J}, \mathcal{K})=\operatorname{eig}(\mathcal{J})$ where $\mathcal{K}$ reduces to an identity matrix. In [32], the imaginary eigenvalues of $\mathcal{J}$ is computed via multi-shift Arnoldi iterations. This algorithm first builds a $p$-dimensional orthogonal basis

$$
V_{p}=\left[v_{1}, v_{2}, \cdots, v_{p}\right]
$$

of the Krylov subspace

$$
\left\{v_{1},(\mathcal{J}-\theta I)^{-1} v_{1}, \cdots,(\mathcal{J}-\theta I)^{-(p-1)} v_{1}\right\}
$$

$(\theta \in j \mathbb{R})$ with a randomly generated initial vector $v_{1} \neq 0$. After that, a $p \times p$ Hessenberg matrix $\mathcal{H}_{p}$ is constructed by

$$
\mathcal{H}_{p}=V_{p}^{H}(\mathcal{J}-\theta I)^{-1} V_{p} .
$$

Subsequently a few largest eigenvalues $\hat{\lambda}_{j}$ of the lowdimension Hessenberg matrix can be used to approximate the true eigenvalues $\lambda_{j}$ of $\mathcal{J}$ close to $\theta$ by

$$
\lambda_{j} \approx \theta+\hat{\lambda}_{j}^{-1}
$$

With an error control all $\lambda$ 's close to $\theta$ can be accurately computed. To compute all possible purely imaginary eigenvalues of $\mathcal{J}, \theta$ should be shifted along the imaginary axis on the upper half plane. In [32], a bisection scheme is adopted in the interval $\left(0, \theta_{\max }\right)$ to fix the shifted parameters, such that all imaginary eigenvalues close to $\theta$ 's are accurately found (see [32] for the details about error control and bisection scheme). Here $\theta_{\max }$ is an estimated largest-magnitude imaginary eigenvalue, which can be easily approximated by sparse power iteration. In this section, we focus on how to extend this approach to the matrix pencil $(\mathcal{J}, \mathcal{K})$. Note that $\tilde{A}$ is assumed to be diagonal in [32], which is waived here.

Assume $\lambda \in \operatorname{eig}(\mathcal{J}, \mathcal{K})$, the nonsingularity of $\mathcal{K}$ implies $\operatorname{eig}(\mathcal{J}, \mathcal{K})=\operatorname{eig}\left(\mathcal{K}^{-1} \mathcal{J}\right)$. To apply the above fast eigenvalue solver, the main bottleneck lies in computing $\left(\mathcal{K}^{-1} \mathcal{J}-\theta I\right)^{-1} v_{i}$. First, $\left(\mathcal{K}^{-1} \mathcal{J}-\theta I\right)$ and its inverse are not sparse, which renders the matrix-vector production inefficient. Second, the 
TABLE I

Total CPU TIMES IN DS INDEX CHECK

\begin{tabular}{l|c|c|c|c|c|c|c}
\hline System & System & \multicolumn{4}{|c|}{ Nos. of Nonzeros in } & \multicolumn{2}{c}{ Time (s) } \\
\cline { 3 - 8 } Order & Index & $A_{0}$ & $E_{0}$ & $E_{1}$ & $E_{2}$ & SVD & LU \\
\hline 480 & 2 & $1346(0.584 \%)$ & $18290(7.94 \%)$ & $19001(8.25 \%)$ & $71521(31.04 \%)$ & 2.411 & 0.8125 \\
578 & 2 & $1694(0.507 \%)$ & $25432(7.61 \%)$ & $26601(7.96 \%)$ & $224130(67.09 \%)$ & 4.506 & 2.8231 \\
980 & 2 & $2872(0.299 \%)$ & $83568(8.70 \%)$ & $85339(8.89 \%)$ & $666669(69.42 \%)$ & 20.359 & 12.80 \\
1232 & 2 & $3634(0.239 \%)$ & $3608(0.238 \%)$ & $3610(0.238 \%)$ & $5504(0.363 \%)$ & 29.250 & 0.0469 \\
10082 & 2 & $30184(0.030 \%)$ & $29568(0.029 \%)$ & $29570(0.029 \%)$ & $31464(0.031 \%)$ & fail & 0.0938 \\
10913 & 2 & $54159(0.045 \%)$ & $35904(0.030 \%)$ & $36087(0.030 \%)$ & $5385968(4.522 \%)$ & fail & 93.28 \\
\hline
\end{tabular}

matrix inversions involved are prohibitively expensive, timeconsuming and unstable for large-scale systems. Therefore, fast, sparse, and stable matrix operations are needed. Since $\mathcal{K}^{-1} \mathcal{J}-\theta \boldsymbol{I}=\mathcal{K}^{-1}(\mathcal{J}-\theta \mathcal{K})$, the matrix-vector production in each iteration can be expressed as

$$
\left(\mathcal{K}^{-1} \mathcal{J}-\theta I\right)^{-1} v_{i}=(\mathcal{J}-\theta \mathcal{K})^{-1} \mathcal{K} v_{i}
$$

By the matrix inverse lemma [34], we have

$$
\begin{aligned}
& (\mathcal{J}-\theta \mathcal{K})^{-1} \mathcal{K} v_{i} \\
& =\left(\left[\begin{array}{ll}
M_{\theta} & \\
& -M_{\theta}^{H}
\end{array}\right]-\left[\begin{array}{c}
\tilde{B} \\
-\tilde{C}^{T}
\end{array}\right]\left(\tilde{D}+\tilde{D}^{T}\right)^{-1}\left[\begin{array}{cc}
\tilde{C} & \tilde{B}^{T}
\end{array}\right]\right)^{-1} w_{i} \\
& =\left[\begin{array}{cc}
M_{\theta}^{-1} & \\
& -M_{\theta}^{-H}
\end{array}\right] w_{i}+\left[\begin{array}{c}
M_{\theta}^{-1} \tilde{B} \\
M_{\theta}^{-H} \tilde{C}^{T}
\end{array}\right] \times \\
& \left(\tilde{D}+\tilde{D}^{T}-\tilde{C} M_{\theta}^{-1} \tilde{B}-\tilde{B}^{T} M_{\theta}^{-H} \tilde{C}^{T}\right)^{-1} \\
& {\left[\begin{array}{ll}
\tilde{C} M_{\theta}^{-1} & -\tilde{B}^{T} M_{\theta}^{-H}
\end{array}\right] w_{i}}
\end{aligned}
$$

where $M_{\theta}=\tilde{A}-\theta \tilde{E}, w_{i}=\mathcal{K} v_{i}$. We first denote $w_{i 1}=w_{i(1: n)}$ and $w_{i 2}=w_{i(n+1: 2 n)}$. Making use of the sparsity of $M_{\theta}$, (29) can be implemented as follows.

1) Perform a sparse LU decomposition on the $n \times n$ matrix

$$
M_{\theta}=L_{\theta} U_{\theta}
$$

then the sparse $\mathrm{LU}$ of $M_{\theta}^{H}$ is readily obtained as $M_{\theta}^{H}=$ $U_{\theta}^{H} L_{\theta}^{H}$.

2) Compute the following vectors (or vector-like narrow matrices)

$$
\begin{aligned}
& x_{1}=U_{\theta}^{-1} L_{\theta}^{-1} w_{i 1} \\
& x_{2}=-L_{\theta}^{-H} U_{\theta}^{-H} w_{i 2} \\
& x_{3}=U_{\theta}^{-1} L_{\theta}^{-1} \tilde{B} \\
& x_{4}=L_{\theta}^{-H} U_{\theta}^{-H} \tilde{C}^{T}
\end{aligned}
$$

by forward/backward iteration. Since $L_{\theta}$ and $U_{\theta}$ obtained by sparse LU are normally sparse, the above linear system solution can be as cheap as $O(n)$.

3) Finally, the inverse iteration can be computed by

$$
\begin{aligned}
& \left(\mathcal{K}^{-1} \mathcal{J}-\theta I\right)^{-1} v_{i}=\left[\begin{array}{l}
x_{1} \\
x_{2}
\end{array}\right]+\left[\begin{array}{l}
x_{3} \\
x_{4}
\end{array}\right] \\
& \times\left(\tilde{D}+\tilde{D}^{T}-\tilde{C} x_{3}-\tilde{B}^{T} x_{4}\right)^{-1}\left(\tilde{C} x_{1}+\tilde{B}^{T} x_{2}\right) .
\end{aligned}
$$

Due to the low dimension, the matrix inversion in this step is trivial.

In the above procedures, the total computation and storage are low since only one $n \times n$ sparse LU factorization is needed, and only sparse upper/lower triangular matrices and vectors are stored.

To this end, the multi-shift Arnoldi algorithm can be used to compute all generalized eigenvalues of $(\mathcal{J}, \mathcal{K})$ close to (or located on) the imaginary axis. The error control and bisection methods are the same as those of the original algorithm [32], which is omitted here. It is clear that compared with standard Hamiltonian method, the GHM-based method avoids largescale matrix inversion and preserves system sparsity.

\section{Complexity Analysis}

DS passivity test by the extended positive real lemma in [19] via solution of LMIs is impractical for large systems due to its $O\left(n^{6}\right)$ complexity [2] where $n$ is the dimension of the state vector in (1). Also, direct transformation to the Weierstrass canonical form (2) can be numerically unstable and expensive. For example, the generalized upper triangular form (GUPTRI) algorithm requires essentially $\geq 3 \times \mathrm{SVD}$, $\geq 2 \times \mathrm{QR}$, and $1 \times \mathrm{QZ}$ decompositions to produce a generalized Schur form, followed by solving an additional generalized Sylvester equation to reach the Weierstrass form, all costing expensive $O\left(n^{3}\right)$ work [35], [36]. Consequently, the more efficient SHH transformation technique is proposed [2] to decouple the original DS into its proper and improper subsystems. A careful inspection reveals that the major SHH steps call for 3 times SVD and $2 \times \mathrm{QR}$ factorizations, plus the optional solution of an algebraic Riccati equation (ARE) and a Lyapunov equation if the proper part is to be explicitly extracted. We are also aware of an $O\left(n^{3}\right)$ DS decoupling procedure in [22] and [23], which costs at least $3 \times$ URV (similar to $\mathrm{SVD}), 1 \times \mathrm{QR}$, and one generalized Schur decomposition, and therefore, has comparable complexity to the SHH test (but the lack of implementation details in [22] and [23] allow no further conclusion about its actual speed). A similar procedure in [21], based on the Van Dooren technique [37], also suffers from multiple SVDs, numerically sensitive sub-matrix annihilations and the costly solution of an ARE. Finally, the recent DS decoupling approach, though not exactly related to passivity test, utilizes the iterative disk function method [38]. But again it requires 10-20 iterations of QR factorizations (on $2 n \times n$ matrices) for convergence, followed by special subspace extraction procedures. Another demanding requirement in all 
the above mentioned procedures, where SVD/QR of dense matrices are necessary, is the $O\left(n^{2}\right)$ memory requirement.

After DS decoupling, either by the disk function, Weierstrass-form or SHH decoupling, the improper subsystem (specifically the residual $M_{1}$ ) is then checked for positive semidefinite which is fast and trivial. On the other hand, the proper-part passivity can be checked with standard regularsystem passivity tests such as the Hamiltonian matrix eigenvalue test of $O\left((2 n)^{3}\right)$ work [16], or the faster but less reliable frequency sweeping test of $O\left(\mathrm{~m}^{3}\right)$ to $O\left(n^{3}\right)$ work [17] depending on the availability of closed-form rational transfer function (e.g., a possible case where analytical transfer matrix exists is when the state-space matrices are obtained from vector fitting as in [17], but this is not always the case if the initial model is not built from rational fitting). In this paper, we directly perform GHM [28], [29] passivity test on the DS-form proper subsystem, which is implemented by the fast multi-shift Arnoldi iteration based algorithm. Assume $t$ shift parameters in the interval $\left(0, \theta_{\max }\right)$ are used to compute all eigenvalues close to the imaginary axis, then $t$ sparse LUs are needed. For each shift, several to tens of Krylov subspace iterations are needed to approximate the neighborhood eigenvalues, which depends on how dense these eigenvalues are distributed. In practice, only a small number of eigenvalues are distributed around the imaginary axis, so usually $t$ is in the tens. Since the expensive matrix inversion is avoided and sparsity is preserved, this method is much more efficient than standard Hamiltonian test on the standard state-space model converted from (15).

It is seen that all the above DS decomposition/decoupling approaches require at least SVD or iterative QR operations which can of course be employed for the matrix chain formation and projector construction in Section III (e.g., [1]). Nonetheless, a key advantage of the projector-based DS passivity test is that the singularity test and spectral projector formulation can all be accomplished with the much cheaper LUtype decomposition, with $\approx O\left(2 n^{3} / 3\right)$ work versus $\approx O\left(20 n^{3}\right)$ in SVD or $\approx O\left(2 n^{3}\right)$ in QR for general dense matrices [34], as described in Section V-A. If sparsity and low-rank operations are further exploited, the projector can be realized with $O\left(n^{2}\right)-$ $O\left(n^{3}\right)$ work and $O(n)$ storage, depending on the sparsity extent. In fact, for index- 2 problems, the main load in the projector construction involves only $5 \times$ sparse LU factorizations (viz. 3 rank tests and 2 nullspace computations). Subsequently, sparsity-aware LU factorization has much reduced memory storage requirement than the SHH or transformations to the Weierstrass form, where dense SVD, QR or matrix equation solves are generally unavoidable. Together with the fast GHM passivity test in Section V-B, the proposed DS passivity test features remarkable computational savings and is applicable to large systems, as will be seen in the following numerical examples.

\section{NUMERICAL EXAMPLES}

In this section, some practical DS benchmarks are tested for passivity to demonstrate the effectiveness of the proposed projector framework. These examples are mostly taken from [39] except the order-1232 and order-10082 ones which are RC
TABLE II

CPU Times ExCluding Proper-PART PASSIVITY CHECK

\begin{tabular}{l|c|c|c}
\hline System & \multicolumn{3}{|c}{ CPU Time (s) } \\
\cline { 2 - 4 } Order & Proposed & SHH & Weierstrass \\
\hline 480 & 1.73 & 9.53 & 10.92 \\
578 & 3.26 & 15.90 & 18.50 \\
980 & 14.84 & 75.83 & 95.41 \\
1232 & 0.0625 & 132.2 & 801 \\
10082 & 0.1563 & fail & fail \\
10913 & 159.68 & fail & fail \\
\hline
\end{tabular}
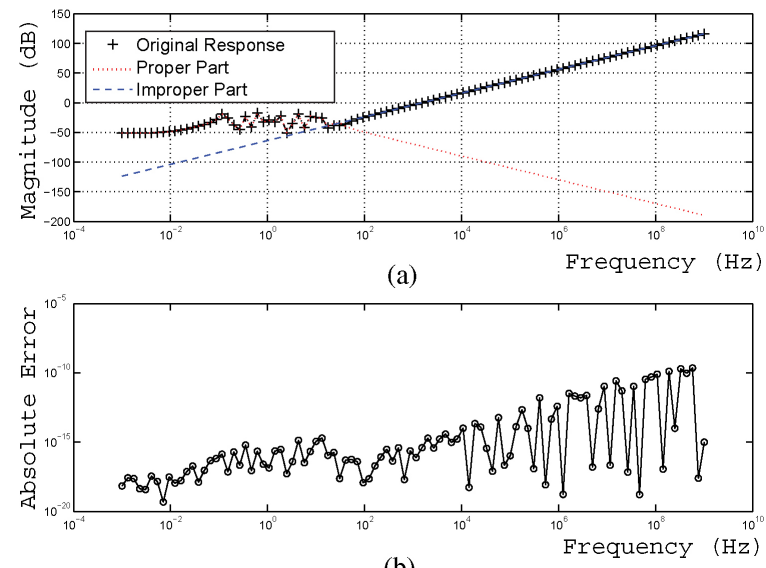

(b)

Fig. 2. (a) Bode plots of the original, proper and improper-subsystem responses. (b) Error between the original system and the sum of decomposed subsystems.

networks. The order-480 system is a patch antenna model from partial element equivalent circuit (PEEC) modeling, while other higher order examples are MNA benchmarks. All MNA benchmarks are passive, while the PEEC model is nonpassive. Nonpassive PEEC models may be caused by poor meshing generation, inadequate numerical integration, matrix sparsification or inappropriate geometrical discretization, which is discussed in detail by Ekman et al. [40]. All codings are done in MATLAB and executed on a $2.66 \mathrm{GHz}$ PC with $2 \mathrm{~GB}$ memory.

We begin by timing the index tests based on checking the nullities of $E_{1}$ and $E_{2}$ [via (19)] of these benchmarks, which forms the initial step in the proposed DS passivity test. Two approaches are contrasted, namely, the standard SVD and the (sparse) LU singularity test discussed in Section V-A. Expectedly, Table I shows that the LU way is much more efficient than the SVD approach even though in some cases the sparsity of $E_{2}$ may not be significant due to the "fill-ins" by matrix chain formation.

Next, the proposed DS passivity test is compared to the SHH [2] and the Weierstrass tests in terms of proper and improper part decoupling, where in the Weierstrass approach the public routine GUPTRI [35] and the SLICOT [41] generalized Sylvester equation solver slgesg (both calling compiled Fortran routines) are employed. The extended LMI test in [19] has been shown by [2] to be computationally impractical and is, therefore, not included here. Table II again demonstrates the 


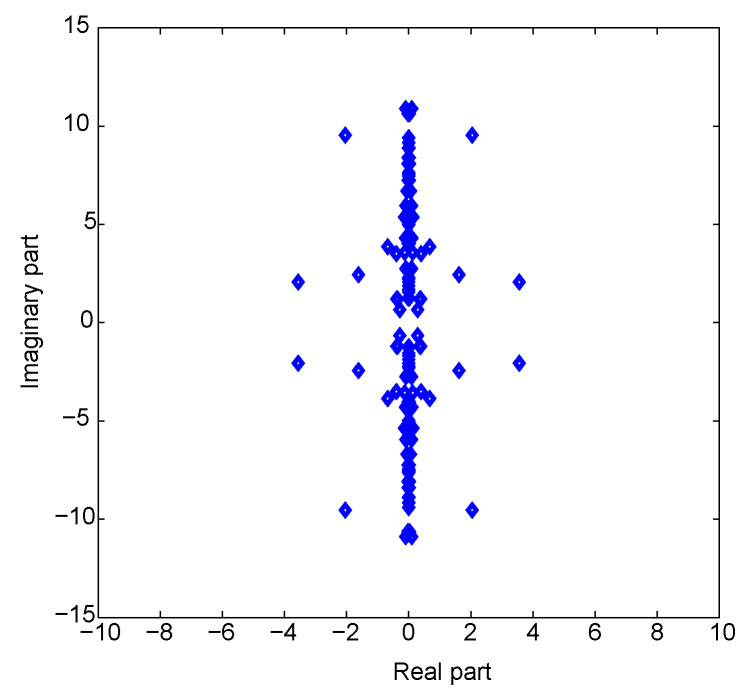

Fig. 3. Eigenspectrum of the generalized Hamiltonian matrix pencil $(\mathcal{J}, \mathcal{K})$ for the PEEC model. Only the results close to the imaginary axis are plotted.

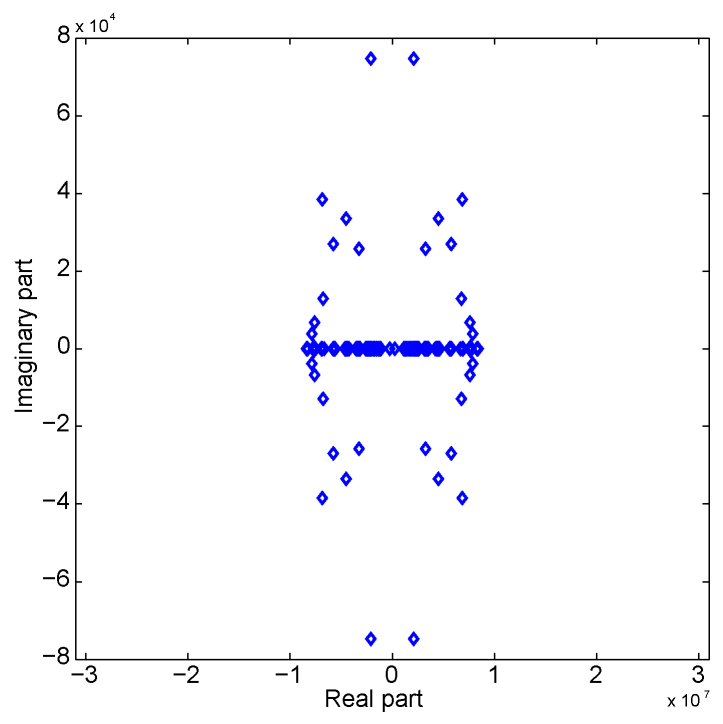

Fig. 4. Eigenspectrum of the generalized Hamiltonian matrix pencil $(\mathcal{J}, \mathcal{K})$ for the order-980 RLC model. Only the results close to the imaginary axis are plotted.

superiority of the projector approach, owing to the techniques presented in Section V-A and the reasonings in Section V-C. It is noted that the much longer time in the Weierstrass column in the order-1232 case is due to the generation of complex entries by GUPTRI which has also been observed in [2]. In the same example, the speed gain from the projector approach is especially impressive (namely, four orders faster) due to the strong sparsity of the RC network. This further highlights the efficiency of the projector approach and in particular its suitability for electrical networks commonly used in very large-scale integrated interconnect/package modeling. To confirm the accuracy of the spectral projector decoupling, we decompose the $10913 \times 10913$ example which is a 9input-9-output system. The proper and improper parts are extracted by additive decomposition (see Section IV). Fig. 2 shows the port- 2 to port- 2 decoupled responses, wherein the
TABLE III

CPU TIMES OF FULL-SIZE GHM AND FAST GHM PASSIVITY TESTS ON THE PROPER SUBSYSTEM

\begin{tabular}{l|c|c|c}
\hline System Order & Full-Size GHM & Fast GHM & Speedup \\
\hline 480 & $21.3 \mathrm{~s}$ & $7.3 \mathrm{~s}$ & 2.9 \\
980 & $83.7 \mathrm{~s}$ & $15.2 \mathrm{~s}$ & 5.6 \\
10913 & fail & $11 \mathrm{hrs} \mathrm{37min}$ & N/A \\
\hline
\end{tabular}

error is in the order of $10^{-10}$ (and similarly for other portto-port responses). After the decoupling, the proper part can be tested by SHH, Weierstrass and the proposed fast GHM method.

We continue to show the applicability of the projectorbased DS passivity test at higher system orders than those considered in [2] and [1], where the highest order is 800 and the SHH and Weierstrass tests consume about 350-600 s on a standard PC. The multi-shift Arnoldi iteration based GHM test in Section V-B is adopted. We begin with the order-480 PEEC example which is a single-input-single-output DS. The projector-based decomposition shows that it is impulse-free. Its proper-part is then tested by the proposed fast GHM test. Fig. 3 shows that some eigenvalues of $(\mathcal{J}, \mathcal{K})$ locate on the imaginary axis, implying that this DS is nonpassive. The next example is the order-980 case originating from a 4-input-4output RLC network, which does not contain an improper part either. The proper-part admittance transfer function is also checked by the proposed fast GHM test. Fig. 4 shows the corresponding passivity test matrix pencil does not contain any purely imaginary generalized eigenvalues. This confirms the model is passive which is expected due to the RLC nature of the example. The third example is the 9-input-9-output order10913 MNA example. The improper part contains only $s M_{1}$ where $M_{1}$ is a diagonal $9 \times 9$ positive definite matrix. As for its proper part, Fig. 5 plots the GHM test results which confirm its passivity, whereas the SHH, Weierstrass transformations and full-size GHM test simply fail at such high orders. In [28] and [29], the superiority of GHM over SHH and Weierstrass tests has been illustrated. We further show the speedup of the proposed fast GHM over the full-size GHM in Table III. The CPU timing shows the speedup of the proposed Arnoldi iteration based GHM (fast GHM) is faster than full-size GHM, and the speedup is more significant as problem size grows. Due to the expensive storage requirement, full-size GHM is not applicable to large sparse systems, but the proposed fast GHM algorithm can still be used to characterize passivity.

\section{REMARKS}

1) Compared to the Weierstrass-form or SHHtransformation DS passivity tests, the proposed algorithm is conceptually simple and straightforward. As described in Section V-C, former DS passivity tests all involve expensive SVD or QR operations for subsystem decoupling, while the projector approach only calls for the much cheaper LU factorizations and much reduced memory requirement when sparsity is exploited. To the knowledge of the authors, this 


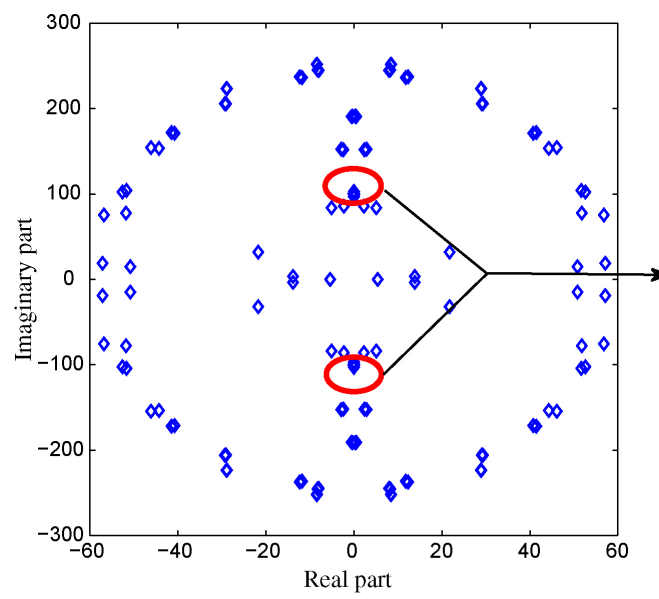

(a)

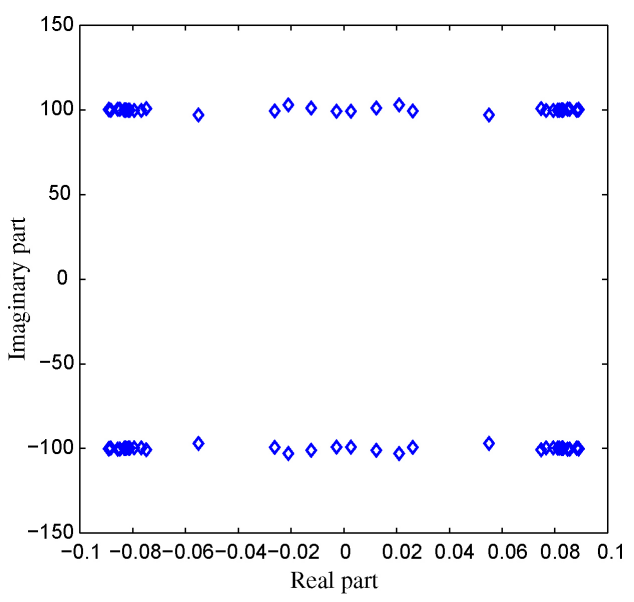

(b)

Fig. 5. Passivity test results for the order-10913 DS model. (a) Eigenspectrum of the generalized Hamiltonian matrix pencil ( $\mathcal{J}, \mathcal{K}$ ). Only the results close to the imaginary axis are plotted. (b) Zoom-in of the eigenvalues with small real parts [circled in (a)].

projector-based passivity test flow for DSs is by far the fastest one reported in the literature.

2) Along the same line, the speed of the proposed projector construction (either explicitly or implicitly as in [31]) is dependent on the sparsity degree and pattern. In many real-world problems, $E_{0}$ and $E_{1}$ are highly sparse and, therefore, the LUQ decomposition [33] is fast. If $E_{2}$ is also sparse enough, the speedup can be drastic as observed in the order-1232 and 10082 examples in Table I. Moreover, the nullities of practical $E_{0}$ or $E_{1}$ are usually low which lead to efficient low-rank formulation of canonical projectors and, therefore, the spectral projector [see (21) and (22)]. This also reinforces the advantageous use of projector techniques in analyzing real-world DSs.

3) Existing DS passivity tests [2], [19], [20], [22], [23] all assume a minimal (i.e., completely controllable and observable) initial DS, otherwise a pre-processing is done to remove the uncontrollable/unobservable states for minimal realization (e.g., by the $O\left(n^{3}\right)$ algorithm in [10] or [2] which essentially reduces a DS into its Kalman canonical form using orthogonal transformations). However, such pre-processing is expensive and destroys sparsity of the system. In fact, Theorem 2 holds under the only assumption of regularity, and what we really require in the DS is the infinite minimality equivalent to $\operatorname{rank}\left[\begin{array}{cc}E & B\end{array}\right]=\operatorname{rank}\left[\begin{array}{ll}E^{T} & C^{T}\end{array}\right]=n$ [19] which determines the highest power of $s$ in (5). Such infinite minimality can be obtained by running the reduction algorithms [2], [10], [21] only partially and is, therefore, much cheaper to achieve than full minimality. Consequently, the minimality assumption in the proposed test is the least restrictive one among existing tests.

4) By virtue of the explicit formulation of regular state spaces for the proper and improper subsystems in Section IV, further processing can be readily exercised. In particular, standard state-space model order reduction
(MOR) and/or passivity enforcement procedures [3], [16], [17], are all reusable on the extracted proper part $G_{p}(s)$. Perturbation of the improper part $G_{\infty}(s)$ into a positive semidefinite first-order residue matrix $M_{1}$, due to its small size (viz. $m \times m$ ), is also trivial.

5) In [28], [29], GHM is directly performed on DS models for passivity check. However, it terminates if the improper part is nonpassive, and then the passivity of the proper subsystem cannot be verified. With the spectral projector technique, the proper part can still be checked regardless of the passivity of the improper part.

\section{CONCLUSION}

This paper has presented a canonical projector-based passivity test for DS models commonly found in circuit modeling. Compared to the existing LMI and SHH transformation approaches for testing DS passivity, the proposed test is theoretically straightforward and exhibits simple codings and superior computational speed. The index of a DS comes at an early stage which immediately reveals the possible passivity of a DS. Fast sparse LU-based construction of the spectral projector then permits efficient decomposition of the DS into its proper and improper subsystems, whose individual passivity can be efficiently evaluated with existing passivity check techniques. State spaces for these subsystems have also been explicitly formulated for utilization in subsequent MOR and/or passivity enforcement. A sparse LU factorization approach to projector construction, together with a fast GHM algorithm based on multi-shift Arnoldi iteration, have made possible the efficient passivity test of high-order DSs unamendable before.

\section{ACKNOWLEDGMENT}

The authors would like to thank Dr. T. Stykel for her valuable comments and suggestions to the content of this paper, as well as the pointers to additional DS passivity tests in the literature. They are also grateful to Prof. L. Daniel for 
the discussion about the passivity in electromagnetic (EM) modeling.

\section{APPENDIX A}

\section{MATRIX CHAIN WITH CANONICAL PROJECTORS}

We begin by making the projector in $(12 \mathrm{~b})$ canonical. This is done by replacing $Q_{1}$ with

$$
Q_{1}^{\prime}:=Q_{1} E_{2}^{-1} A_{1} \text {. }
$$

To see $Q_{1}^{\prime}$ is a valid projector onto $\operatorname{ker} E_{1}$, we note that $Q_{1}^{\prime} Q_{1}=Q_{1} E_{2}^{-1} A_{1} Q_{1}=Q_{1} E_{2}^{-1} E_{2} Q_{1}=Q_{1}$ and $Q_{1} Q_{1}^{\prime}=$ $Q_{1}^{\prime}$, which implies $Q_{1}^{\prime}\left(=Q_{1}^{\prime 2}\right)$ is a projector onto the same range as $Q_{1}$. To show $Q_{1}^{\prime}$ and $Q_{0}$ are admissible, we have $Q_{1}^{\prime} Q_{0}=Q_{1} E_{2}^{-1} A_{1} Q_{0}=Q_{1} E_{2}^{-1} A_{0} P_{0} Q_{0}=0$. Using $Q_{1}^{\prime}$ in place of $Q_{1}$, (III-A) is updated to

$$
\begin{gathered}
E_{1}:=E_{0}+A_{0} Q_{0} \text { and } A_{1}:=A_{0} P_{0} \\
E_{2}^{\prime}:=E_{1}+A_{1} Q_{1}^{\prime} .
\end{gathered}
$$

Note that Theorem 2 again guarantees $E_{2}^{\prime}$ to be nonsingular. Now to verify the canonicity of $Q_{1}^{\prime}$ in $(34 \mathrm{~b})$, we make use of the property in (11) to get $E_{2}^{\prime}=E_{2}\left(I+Q_{1}^{\prime} P_{1}\right)$ and have

$$
\begin{aligned}
Q_{1}^{\prime} E_{2}^{\prime-1} A_{1} & =Q_{1}^{\prime}\left(I-Q_{1}^{\prime} P_{1}\right) E_{2}^{-1} A_{1} \\
& =Q_{1}^{\prime} Q_{1} E_{2}^{-1} A_{1}=Q_{1} E_{2}^{-1} A_{1}=Q_{1}^{\prime}
\end{aligned}
$$

thus completing the proof. Next, we set

$$
Q_{0}^{\prime}:=Q_{0} P_{1}^{\prime} E_{2}^{\prime-1} A_{0} .
$$

Noting $A_{0} Q_{0}=E_{2}^{\prime} Q_{0}$, it follows that $Q_{0}^{\prime 2}=Q_{0}^{\prime}$ is a projector onto ker $E_{0}$. Recompute (34a) with this new projector to give

$$
E_{1}^{\prime}:=E_{0}+A_{0} Q_{0}^{\prime} \text { and } A_{1}^{\prime}:=A_{0} P_{0}^{\prime} .
$$

Again, from (11), $E_{1}^{\prime}=E_{1}\left(I+Q_{0}^{\prime} P_{0}\right)$ and it turns out $Q_{1}^{\prime}$ is also a projector onto ker $E_{1}^{\prime}$, namely

$$
\begin{aligned}
E_{1}^{\prime} Q_{1}^{\prime} & =E_{1}\left(I+Q_{0}^{\prime} P_{0}\right) Q_{1}^{\prime}=E_{1}\left(I+Q_{0} P_{1}^{\prime} E_{2}^{\prime-1} A_{0} P_{0}\right) Q_{1}^{\prime} \\
& =E_{1} Q_{1}^{\prime}+E_{1} Q_{0} P_{1}^{\prime} E_{2}^{\prime-1} A_{1} Q_{1}^{\prime}=0
\end{aligned}
$$

since $A_{1} Q_{1}^{\prime}=E_{2}^{\prime} Q_{1}^{\prime}$. With $Q_{0}^{\prime}$, (A) is further updated as

$$
\begin{aligned}
& E_{1}^{\prime}:=E_{0}+A_{0} Q_{0}^{\prime} \text { and } A_{1}^{\prime}:=A_{0} P_{0}^{\prime} \\
& E_{2}^{\prime \prime}:=E_{1}^{\prime}+A_{1}^{\prime} Q_{1}^{\prime} .
\end{aligned}
$$

Because $Q_{1}^{\prime} Q_{0}=0$, so is $Q_{1}^{\prime} Q_{0}^{\prime}=0$ (i.e., admissible) and

$$
\begin{aligned}
Q_{0}^{\prime} Q_{1}^{\prime} & =Q_{0} P_{1}^{\prime} E_{2}^{\prime-1} A_{0} Q_{1}^{\prime}=Q_{0} P_{1}^{\prime} E_{2}^{\prime-1} A_{0}\left(Q_{0}+P_{0}\right) Q_{1}^{\prime} \\
& =Q_{0} P_{1}^{\prime} E_{2}^{\prime-1} E_{2}^{\prime} Q_{0} Q_{1}^{\prime}+Q_{0} P_{1}^{\prime} E_{2}^{\prime-1} A_{1} Q_{1}^{\prime} \\
& =Q_{0} Q_{1}^{\prime}+Q_{0} P_{1}^{\prime} E_{2}^{\prime-1} E_{2}^{\prime} Q_{1}^{\prime}=Q_{0} Q_{1}^{\prime} .
\end{aligned}
$$

This also implies $P_{0}^{\prime} Q_{1}^{\prime}=P_{0} Q_{1}^{\prime}$ and $A_{1}^{\prime} Q_{1}^{\prime}=A_{1} Q_{1}^{\prime}$, from which a link can be found between $E_{2}^{\prime \prime}$ and $E_{2}^{\prime}$ as

$$
\begin{aligned}
E_{2}^{\prime \prime} & =E_{1}\left(I+Q_{0}^{\prime} P_{0}\right)+A_{1} Q_{1}^{\prime} \\
& =E_{2}^{\prime} P_{1}^{\prime}\left(I+Q_{0}^{\prime} P_{0}\right)+E_{2}^{\prime} Q_{1}^{\prime}=E_{2}^{\prime}\left(I+Q_{0}^{\prime} P_{0}\right) .
\end{aligned}
$$

Still, we need to show both $Q_{0}^{\prime}$ and $Q_{1}^{\prime}$ in (A) are indeed canonical. Utilizing properties (39), (40) and noting that $A_{1}=$ $A_{0} P_{0}=A_{0} P_{0} P_{0}^{\prime}=A_{1}^{\prime}-E_{2}^{\prime} Q_{0} P_{0}^{\prime}$, we get

$$
\begin{aligned}
Q_{0}^{\prime} P_{1}^{\prime} E_{2}^{\prime \prime-1} A_{0} & =Q_{0}^{\prime}\left(I-Q_{1}^{\prime}\right)\left(I-Q_{0}^{\prime} P_{0}\right) E_{2}^{\prime-1} A_{0} \\
& =\left(Q_{0}^{\prime}-Q_{0}^{\prime} Q_{1}^{\prime}-Q_{0}^{\prime} P_{0}\right) E_{2}^{\prime-1} A_{0} \\
& =\left(Q_{0}^{\prime} Q_{0}-Q_{0}^{\prime} Q_{1}^{\prime}\right) E_{2}^{\prime-1} A_{0} \quad \text { [see (39)] } \\
& =Q_{0} P_{1}^{\prime} E_{2}^{\prime-1} A_{0}=Q_{0}^{\prime} \quad[\text { see (36)] } \\
Q_{1}^{\prime} E_{2}^{\prime \prime-1} A_{1}^{\prime} & =Q_{1}^{\prime}\left(I-Q_{0}^{\prime} P_{0}\right) E_{2}^{\prime-1} A_{1}^{\prime} \\
& =Q_{1}^{\prime} E_{2}^{\prime-1}\left(A_{1}+E_{2}^{\prime} Q_{0} P_{0}^{\prime}\right) \\
& =Q_{1}^{\prime} E_{2}^{\prime-1} A_{1}=Q_{1}^{\prime} \quad[\text { see (35)]. }
\end{aligned}
$$

Of course, in practice, only the canonical projectors $Q_{0}^{\prime}$ and $Q_{1}^{\prime}$ are required for computing the spectral projectors, and the matrix chain updates from (III-A) to (A) and then to (A) never need to be computed explicitly.

\section{APPENDIX B}

\section{SPECTRAL PROJECTORS FROM CANONICAL PROJECTORS}

We give a constructive proof for $P_{r}=P_{0}^{\prime} P_{1}^{\prime}$ for the case $\mu=2$. This complements, and is also in contrast to, the indirect argument toward spectral projector presented in [25]. Taking the Weierstrass viewpoint for $E_{0}$ and $A_{0}$ (wherein $N^{2}=0$ ) and assuming canonical $Q_{0}^{\prime}$ and $Q_{1}^{\prime}$, we can easily show

$$
Q_{0}^{\prime}=T^{-1}\left[\begin{array}{cc}
0 & 0 \\
0 & \widehat{Q}_{0}^{\prime}
\end{array}\right] T \text { and } Q_{1}^{\prime}=T^{-1}\left[\begin{array}{cc}
0 & 0 \\
0 & \widehat{Q}_{1}^{\prime}
\end{array}\right] T
$$

where $\widehat{Q}_{0}^{\prime}, \widehat{Q}_{1}^{\prime} \in \mathbb{R}^{(n-q) \times(n-q)}$ project onto $\operatorname{ker} N$ and $\operatorname{ker}(N+$ $\left.\widehat{Q}_{0}^{\prime}\right)$, respectively. Moreover

$$
N_{2}:=N+\widehat{Q}_{0}^{\prime}+\widehat{P}_{0}^{\prime} \widehat{Q}_{1}^{\prime}=N+I-\widehat{P}_{0}^{\prime} \widehat{P}_{1}^{\prime}
$$

is nonsingular due to Theorem 2. By canonicity

$$
\widehat{Q}_{0}^{\prime}=\widehat{Q}_{0}^{\prime} \widehat{P}_{1}^{\prime} N_{2}^{-1}, \quad \widehat{Q}_{1}^{\prime}=\widehat{Q}_{1}^{\prime} N_{2}^{-1} \text { and } \widehat{Q}_{1}^{\prime} \widehat{Q}_{0}^{\prime}=0 .
$$

Next, we prove $\widehat{P}_{0}^{\prime} \widehat{P}_{1}^{\prime}=0$. First

$$
N N_{2}=N^{2}+N \widehat{Q}_{0}^{\prime}+N \widehat{P}_{0}^{\prime} \widehat{Q}_{1}^{\prime}=N \widehat{Q}_{1}^{\prime} .
$$

Post-multiplying $N_{2}^{-1}$ to (44) and using (43) we have $N=$ $N \widehat{Q}_{1}^{\prime}$ or equivalently $N \widehat{P}_{1}^{\prime}=0$. Next, recognizing $\widehat{P}_{0}^{\prime} \widehat{P}_{1}^{\prime}$ is a projector by itself, i.e., $\left(\widehat{P}_{0}^{\prime} \widehat{P}_{1}^{\prime}\right)^{2}=\widehat{P}_{0}^{\prime} \widehat{P}_{1}^{\prime}$, and post-multiplying it to (42), we obtain

$$
N_{2} \widehat{P}_{0}^{\prime} \widehat{P}_{1}^{\prime}=N \widehat{P}_{0}^{\prime} \widehat{P}_{1}^{\prime}=N \widehat{P}_{1}^{\prime}=0 .
$$

Nonsingularity of $N_{2}$ then implies $\widehat{P}_{0}^{\prime} \widehat{P}_{1}^{\prime}=0$. Consequently, the right (spectral) projector $P_{r}$ in (3) is given by

$$
P_{r}=P_{0}^{\prime} P_{1}^{\prime}=T^{-1}\left[\begin{array}{cc}
I_{q} & 0 \\
0 & \widehat{P}_{0}^{\prime} \widehat{P}_{1}^{\prime}
\end{array}\right] T=T^{-1}\left[\begin{array}{cc}
I_{q} & 0 \\
0 & 0
\end{array}\right] T .
$$

To obtain the left projector, canonical projectors are constructed from the matrix chain starting instead with $E_{0}:=E^{T}$ and $A_{0}:=A^{T}$. It is then easily verified that

$$
P_{l}=\left(P_{0}^{\prime} P_{1}^{\prime}\right)^{T}=W\left[\begin{array}{cc}
I_{q} & 0 \\
0 & 0
\end{array}\right] W^{-1} \text {. }
$$




\section{APPENDIX C}

AN OUTLINE OF THE LUQ ROUTINE

Given a sparse $n \times n$ matrix $Z$, the LUQ routine gives

$$
Z=L_{Z}\left[\begin{array}{cc}
U_{Z} & 0 \\
0 & 0
\end{array}\right] Q_{Z}
$$

with $L_{Z}, U_{Z}, Q_{Z}$ being nonsingular and $U_{Z}$ being upper triangular. In the first step of LUQ, $Z$ is factorized by sparse LU with permutation

$$
Z=P L\left[\begin{array}{cc}
U_{1} & U_{2} \\
0 & U_{3}
\end{array}\right] Q .
$$

Here, $P$ and $Q$ are permutation matrices, $L$ is lower triangular, $U_{1}$ is an upper triangular matrix with nonzero diagonal elements, $U_{3} \in \mathbb{R}^{s \times s}$ is a very sparse upper triangular matrix with zero diagonals, and $U_{2}$ is a nonzero matrix. The zero/nonzero diagonal elements can be distinguished by setting a small numerical threshold as adopted in economic SVD in MATLAB. Denoting $L:=\left[\begin{array}{ll}L_{1} & L_{2}\end{array}\right]$ and $Q:=\left[\begin{array}{l}Q_{1} \\ Q_{2}\end{array}\right]$, can be rewritten as

$$
Z=P\left[\begin{array}{ll}
L_{1} & L_{2}
\end{array}\right]\left[\begin{array}{cc}
U_{1} & 0 \\
0 & U_{3}
\end{array}\right]\left[\begin{array}{c}
Q_{1}+U_{1}^{-1} U_{2} Q_{2} \\
Q_{2}
\end{array}\right] .
$$

If $U_{3}$ (which is normally of low dimension if $Z$ has a low nullity) has nonzero elements, we can recursively perform LUQ on this block such that

$$
U_{3}=L_{U 3}\left[\begin{array}{cc}
U_{U 3} & 0 \\
0 & 0
\end{array}\right] Q_{U_{3}}
$$

with $U_{U 3}$ being a nonsingular upper triangular matrix. Then, $L_{Z}, U_{Z}$ and $Q_{Z}$ in (48) can be decided by

$$
\begin{aligned}
L_{Z} & =\left[\begin{array}{ll}
P L_{1} & P L_{2} L_{U 3}
\end{array}\right] \\
U_{Z} & =\left[\begin{array}{cc}
U_{1} & 0 \\
0 & U_{U 3}
\end{array}\right] \\
Q_{Z} & =\left[\begin{array}{c}
Q_{1}+U_{1}^{-1} U_{2} Q_{2} \\
Q_{U 3} Q_{2}
\end{array}\right] .
\end{aligned}
$$

The cost of LUQ mainly comes from the sparse LU of $Z$, since $U_{3}$ is highly sparse and of low dimension. From (C) it is clear that $L_{Z}$ is a product of a permutation matrix and a sparse lower triangular matrix. Nevertheless, $Q_{Z}$ might be full and inaccurate since $U_{1}^{-1}$ is involved. That is why we perform LUQ on $E_{j}^{T}$ [see (19)] to construct the null space of $E_{j}$.

\section{REFERENCES}

[1] N. Wong, "An efficient passivity test for descriptor systems via canonical projector techniques," in Proc. IEEE Design, Autom. Conf., Jul. 2009, pp. 957-962.

[2] N. Wong and C. K. Chu, "A fast passivity test for stable descriptor systems via skew-Hamiltonian/Hamiltonian matrix pencil transformations," IEEE Trans. Circuits Syst. I, vol. 55, no. 2, pp. 635-643, Mar. 2008.

[3] J. R. Phillips, L. Daniel, and L. M. Silveira, "Guaranteed passive balancing transformations for model order reduction," IEEE Trans. Comput.-Aided Design Integr. Circuits Syst., vol. 22, no. 8, pp. 1027 1041, Aug. 2003.

[4] K. E. Brenan, S. L. Campbell, and L. Petzold, Numerical Solution of Initial-Value Problems in Differential Algebraic Equations (Classics in Applied Mathematics), vol. 14, 2nd ed. Philadelphia, PA: SIAM, 1996.
[5] E. Eich-Soellner and C. Führer, Numerical Methods in Multibody Dynamics. Stuttgart, Germany: B.G. Teubner, Jul. 1998.

[6] M. Günther and U. Feldmann, "CAD-based electric-circuit modeling in industry I: Mathematical structure and index of network equations," Surveys Math. Indust., vol. 8, no. 2, pp. 97-129, 1999.

[7] M. Günther and U. Feldmann, "CAD-based electric-circuit modeling in industry II: Impact of circuit configurations and parameters." Surveys Math. Indust., vol. 8, no. 2, pp. 131-157, 1999.

[8] E. L. Yip and R. F. Sincovec, "Solvability, controllability and observability of continuous descriptor systems," IEEE Trans. Automat. Control, vol. 26, no. 3, pp. 702-707, Jun. 1981

[9] L. Dai, Singular Control Systems (Lecture Notes in Control and Information Sciences Series 118). Berlin, Germany: Springer-Verlag, 1989.

[10] A. Varga, "A descriptor systems toolbox for MATLAB," in Proc. IEEE Int. Symp. Comput.-Aided Control Syst. Design, 2000, pp. 150-155.

[11] D. E. Schwarz and C. Tischendorf, "Structural analysis of electric circuits and consequences for MNA," Int. J. Circ. Theor. Appl., vol. 28, no. 2, pp. 131-162, Aug. 2000

[12] I. Balk, "On a passivity of the Arnoldi based model order reduction for full-wave electromagnetic modeling," IEEE Trans. Adv. Packag., vol. 24, no. 3, pp. 304-308, Aug. 2001

[13] A. Mayo and A. Antoulas, "A framework for the solution of the generalized realization problem," Linear Algebra Appl., vol. 425, nos. 2-3, pp. 634-662, Sep. 2007.

[14] S. Lefteriu and A. C. Antoulas, "Modeling multi-port systems from frequency response data via tangential interpolation," in Proc. IEEE Workshop Signal Propagation Interconnects, May 2009, pp. 1-4.

[15] B. Yan, S.-D. Tan, and B. McGaughy, "Second-order balanced truncation for passive-order reduction of RLCK circuits," IEEE Trans. Circuits Syst. II, vol. 55, no. 9, pp. 942-946, Sep. 2008.

[16] S. Grivet-Talocia and A. Ubolli, "A comparative study of passivity enforcement schemes for linear lumped macromodels," IEEE Trans. Adv Packag., vol. 31, no. 4, pp. 673-683, Nov. 2008.

[17] D. Saraswat, R. Achar, and M. S. Nakhla, "Fast passivity verification and enforcement via reciprocal systems for interconnects with large order macromodels," IEEE Trans. VLSI Syst., vol. 15, no. 1, pp. 48-59, Sep. 2007.

[18] Y. Liu and N. Wong, "Fast sweeping methods for checking passivity of descriptor systems," in Proc. IEEE Asia Pacific Conf. Circuits Syst., Dec. 2008, pp. 1794-1797.

[19] R. W. Freund and F. Jarre, "An extension of the positive real lemma to descriptor systems," Optim. Methods Softw., vol. 19, no. 1, pp. 69-87, Feb. 2004.

[20] T. Reis and T. Stykel, "Positive real and bounded rea balancing for model reduction of descriptor systems," Inst. für Mathematik, Tech. Univ. Berlin, Berlin, Germany, Tech. Rep. 2008/25, 2008 [Online]. Available: http://www.math.tu-berlin. de/ stykel/Publications/pr_08_25.pdf

[21] R.-C. Li, "Test positive realness of a general transfer function matrix," Dept. Math., Univ. of Kentucky, Lexington, Tech. Rep. 2000-20, 2004 [Online]. Available: http://www.ms.uky.edu/ math/MAreport/00-20.ps

[22] P. Benner and D. Chu, "A new test for passivity of descriptor systems," Oberwolfach Reports, Mathematisches Forschungsinstitut Oberwolfach, Oberwolfach, Germany, Tech. Rep. 11/2005, Feb. 2005.

[23] D. Chu and R. C. E. Tan, "Algebraic characterizations for positive realness of descriptor systems," SIAM J. Matrix Anal. Appl., vol. 30, no. 1, pp. 197-222, 2008.

[24] E. Griepentrog and R. März, "Basic properties of some differentialalgebraic equations," Zeitschrift Für Anal. Ihre Anwendungen, vol. 8, no. 1 , pp. 25-40, 1989.

[25] R. März, "Canonical projectors for linear differential algebraic equations," Comput. Math. Applicat., vol. 31, no. 4, pp. 121-135, Feb. 1995.

[26] R. März, "Projectors for matrix pencils," Humboldt-Univ. zu Berlin, Berlin, Germany, Tech. Rep. 04-24, 2004 [Online]. Available: http://www.mathematik.hu-berlin.de/publ/pre/2004/P-04-24.ps

[27] W. Cauer, Synthesis of Linear Communication Networks. New York: McGraw-Hill, 1958

[28] Z. Zhang, C. U. Lei, and N. Wong, "GHM: A generalized Hamiltonian method for passivity test of impedance/admittance descriptor systems," in Proc. Int. Conf. Comput.-Aided Design, Nov. 2009, pp. 767-773.

[29] Z. Zhang and N. Wong, "Passivity test of immitance descriptor systems based on generalized Hamiltonian methods," IEEE Trans. Circuits Syst. II, vol. 57, no. 1, pp. 61-65, Jan. 2010.

[30] T. Reis and T. Stykel, "Passivity-preserving balanced truncation for electrical circuits," Inst. für Math., Tech. Univ. Berlin, Berlin, Germany, Tech. Rep. 2008/32, 2008 [Online]. Available: http://www.math.tuberlin.de/ $\sim$ stykel/Publications/pr_08_32.pdf 
[31] T. Stykel, "Low-rank iterative methods for projected generalized Lyapunov equations," Electron. Trans. Numer. Anal., vol. 30, pp. 187-202, Aug. 2008.

[32] S. Grivet-Talocia and A. Ubolli, "On the generation of large passive macromodels for complex interconnect structures," IEEE Trans. Adv. Packag., vol. 29, no. 1, pp. 39-54, Feb. 2006.

[33] P. Kowal. (2006, May). Null Space of a Sparse Matrix. MATLAB Central [Online]. Available: http://www.mathworks.co.uk/matlabcentral/fileexchange/11120

[34] G. Golub and C. V. Loan, Matrix Computations, 3rd ed. Baltimore, MD: The Johns Hopkins Univ. Press, 1989.

[35] J. Demmel and B. Kågström, "The generalized Schur decomposition of an arbitrary pencil A- $\lambda \mathrm{B}$ : Robust software with error bounds and applications I: Theory and algorithms," ACM Trans. Math. Softw. vol. 19 , no. 2, pp. 160-174, Jun. 1993.

[36] M. Gerdin, "Computation of a canonical form for linear differentialalgebraic equations,” Dept. of Electr. Eng., Linköping Univ., Linköping, Sweden, Tech. Rep. LiTH-ISY-R-2602, Apr. 2004.

[37] B. Kågström and P. V. Dooren, "A generalized state space approach for the additive decomposition of a transfer matrix," Int. J. Numer. Lin. Alg. Appl., vol. 1, no. 2, pp. 165-181, 1992.

[38] P. Benner, Scientific Computing in Electrical Engineering 2008 (Mathematics in Industry Series), vol. 14, J. Roos and L. R. J. Costa, Eds. Berlin, Germany: SpringerVerlag, 2009 [Online]. Available: http://www-user.tu-chemnitz. de/ benner/pub/Benner_SCEE2008_revised.pdf

[39] Y. Chahlaoni and P. V. Dooren, "A collection of benchmark examples for model reduction of linear time invariant dynamical systems," SLICOT Working Note, Feb. 2002 [Online]. Available: http://www.icm.tu-bs.de/ NICONET/benchmodred.html

[40] J. Ekman, G. Antonini, A. Orlandi, and A. E. Ruehli, "Stability of PEEC models with respect to partial element accuracy," in Proc. Int Symp. Electromagn. Compatibility, Aug. 2004, pp. 271-276.

[41] P. Benner, V. Mehrmann, V. Sima, S. Van-Huffel, and A. Varga, "SLICOT: A subroutine library in systems and control theory," Appl. Comput. Contr., Signals, Circuits, vol. 1, pp. 499-539, 1999.

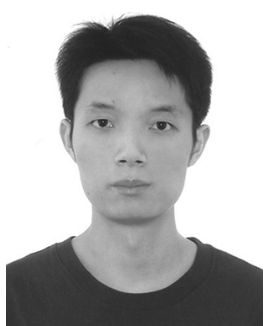

Zheng Zhang (S'09) received the B.Eng. degree in electronic science and technology from Huazhong University of Science and Technology, Wuhan, China. He is currently pursuing the M.Phil. degree from the Department of Electrical and Electronic Engineering, University of Hong Kong, Pokfulam, Hong Kong.

In 2008, he was a Visiting Graduate Student with the Department of Computer Science and Engineering, University of California at San Diego, La Jolla, CA, in 2009. His current research interests include computer-aided design of very large-scale integrated circuits, with emphasis on numerical methods for model order reduction, power grid simulation, and signal integrity analysis.

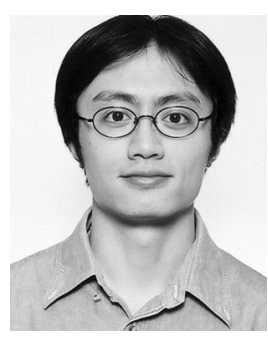

Ngai Wong (S'98-M'02) received the B.Eng. degree with first class honors and the Ph.D. degree, both in electrical and electronic engineering, from the University of Hong Kong, Pokfulam, Hong Kong, in 1999 and 2003, respectively.

From 1997 to 1998, he was an intern with Motorola, Inc., Hong Kong, specializing in product testing. In 2003, he was a Visiting Scholar with Purdue University, West Lafayette, IN. He is Currently an Assistant Professor with the University of Hong Kong. His current research interests include very large-scale integrated (VLSI) model order reduction and simulation, digital filter design, sigma-delta modulators, and optimization problems in communication and VLSI applications. 